\title{
Performance of Hybrid RF/FSO Cooperative Systems Based on Quasicyclic LDPC Codes and Space-Coupled LDPC Codes
}

\author{
Ibrahima Gueye $\mathbb{D}^{D}$, Ibra Dioum $\left(\mathbb{D}\right.$, Idy Diop $\mathbb{D}^{D}$, K. Wane Keita, Papis Ndiaye, Moussa Diallo, \\ and Sidi Mohamed Farssi
}

Department of Computer Science, Polytechnic Institute (ESP), Universite Cheikh Anta Diop, Dakar, Senegal

Correspondence should be addressed to Ibrahima Gueye; gueyeibrahimaig@gmail.com

Received 1 June 2020; Revised 2 November 2020; Accepted 7 December 2020; Published 30 December 2020

Academic Editor: Javier Prieto

Copyright $\odot 2020$ Ibrahima Gueye et al. This is an open access article distributed under the Creative Commons Attribution License, which permits unrestricted use, distribution, and reproduction in any medium, provided the original work is properly cited.

\begin{abstract}
Free space optical (FSO) communication systems provide wireless line of sight connectivity in the unlicensed spectrum, and wireless optical communication achieves higher data rates compared to their radio frequency (RF) counterparts. FSO systems are particularly attractive for last mile access problem by bridging fiber optic backbone connectivity to RF access networks. To cope with this practical deployment scenario, there has been increasing attention to the so-called dual-hop (RF/FSO) systems where RF transmission is used at a hop followed by FSO transmission to another. In this article, we study the performance of cooperative transmission systems using a mixed RF-FSO DF (decode and forward) relay using error-correcting codes including QC-LDPC codes at the relay level. The FSO link is modeled by the gamma-gamma distribution, and the RF link is modeled by the Additive White Gaussian Noise (AWGN) model. Another innovation in this article is the use of cooperative systems using a mixed FSO/RF DF relay using quasicyclic low-density parity check (QC-LDPC) codes at the relay level. We also use the spacecoupled low-density parity check (SC-LDPC) codes on the same scheme to show its importance in cooperative optical transmission but also in hybrid RF/FSO transmission. The latter will be compared with QC-LDPC codes. The use of mixed $\mathrm{RF} / \mathrm{FSO}$ cooperative transmission systems can improve the reliability and transmission of information in networks. The results demonstrate an improvement in the performance of the cooperative RF/FSO DF system based on QC-LDPC and SC-LDPC codes compared to RF/FSO systems without the use of codes, but also to the DF systems proposed in the existing literature.
\end{abstract}

\section{Introduction}

Radiofrequency (RF) communication systems have advantages such as low power with a good operating range and alongside these, here the line of sight (LOS) is not mandatory for communication. However, the problem is a lack of licensed spectrum with insufficient and also expensive data rates. On the contrary, free space optical (FSO) communication systems have obtained the attention of convincing research due to its supremacy in the unlicensed optical spectrum, high bandwidth, high capacity, robustness to electromagnetic interference (EM), easy installation, and highspeed data. Despite the advantages of FSO communication, there are limiting factors that play a major role. In general, over a distance of $1 \mathrm{~km}$, there is a significant deterioration in the quality of the signal represented in the FSO communication system, so communication is either the tween source
(S) and the destination (D) is practically impossible. These main limiting factors are the atmospheric turbulence which causes scintillation and the other is the pointing error [1]. This turbulence is nothing but due to the fluctuation of the refractive index of the atmosphere due to the inhomogeneity of the temperature and the pressure of the air particle. Pointing error is introduced due to dynamic wind load, low thermal expansion earthquake, which causes the tilting of the line of sight (LOS) between the transmitter and the receiver. This pointing error generally occurs in urban areas. Therefore, the mixed RF-FSO technique is used to take the bene crises of both technologies. Therefore, to deploy this technique in practice, we switched to the relay technique.

Cooperative communication is an active area of research because of its ability to improve reliability and extend the coverage of wireless networks while using infrastructure existing. Cooperation techniques have been widely studied 
in the context of free space in optical communication systems (FSO) as a means of mitigating the effects of limiting turbulence induced by atmospheric scintillation [2]. The authors [3] proposed cooperative relay assistance of the FSO framework, such as terrestrial link networks. They also studied in [3] the feasibility of the FSO cooperative communication system $5 \mathrm{G}$ land link framework. A parallel FSO multihop system architecture to make the most of the use of network resources, which has been shown to be superior in increasing reliability and throughput is introduced [4]. The authors [3] consider such a cooperative multihop FSO system with DF parallel relays in the channel state information (CSI) is available for all FSO nodes. They focused on maximizing network throughput and promoting the reliability of transmission in transmission networks. They also proposed a relay selection algorithm in which the back pressure theory is used for promoting flow. Gueye et al. [5] proposed two cooperative schemes to decode and transmit- (DF-) based errorcorrecting codes particularly LDPC codes, namely case I, which involves choosing parallel relays; the transmitting node transmits the data to the receiving node and to a relay node which in turn forwards the data to the receiving node. This form of transmission acts as a distributed network of antennas and is considered a cooperative approach in diversity; case II: the multihop relay is to extend the range of a transmission with a limited transmission range. In this approach, the signal passes from one relay node to another in series. Their results show that, compared to the traditional transmission mode, their cooperative FSO systems make it possible to attenuate the fading induced by atmospheric turbulence but also to protect information.

To improve network reliability and improve coverage, it is important to use relays in both the RF and FSO networks. Mixed systems relaying RF-FSO have been widely discussed in the literature. In the literature, there has been a growing interest in how FSO and RF links can be used in a doublehop configuration to support link uplink [6] and downlink transmissions [7]. In order to provide a general framework for analyzing the performance of mixed FSO-RF systems, the authors [8] considered a dual-hop system where the FSO and RF links, respectively, experience Generalized Double Gamma (DGG) and extended Generalized-K (EGK) fading, and their model works well in different turbulence situations (i.e., weak, moderate, and strong). In [9], a similar mixed RF-FSO DH system using a decode and forward- (DF-) based relay system was studied. In [10], a DF-based RF-FSO mixed system is considered with a single multiple input-output (SIMO) RF source-relay links and a mono-mono-input-output (SISO) FSO relay at destination link. In [11], the authors analyzed the performance of a switching-based FSO/RF hybrid transmission in a $\mathrm{DH}$ scenario with combining selection (SC). In [12], the authors consider a mixed RF-FSO system with a double-hop AF relay in which a partial relay selection scheme was used to select the best relay. They also considered that the RF channels are under constraints and that Rayleigh shares spectrum, while the FSO channel is supposed to follow the gamma-gamma distribution.

It is well known that circumference plays an important role in the design of low-density parity check (LDPC) codes
[13]. In general, LDPC codes of random type are constructed by means of a computer search, for example, a progressive growth algorithm (PEG) [14], under certain rules which can ensure good values of circumference. Like another type of LDPC codes, structured codes constructed from algebraic tools, such as combinatorial design [15], finite field [16], graph theory [17], and finite geometry [18], naturally have a good circumference. Numerical results show that these two classes of well-designed LDPC codes perform well. The $1 \mathrm{~s}$ in the parity check matrix of the random codes is distributed irregularly, which interferes with their hardware implementation. Hence, the structured codes are attracting more and more attention in academia and industry. Quasicyclic (QC) LDPC codes [19] are an important class of structured codes and have the advantages of iterative decoding convergence, good performance in cascades and error floor areas, and the implementation decoding [20] and coding [21]. It is well known that the circumference of fully connected QC-LDPC codes is a maximum of 12 [19].

To the best of the authors' knowledge, research on the aspect of RF/FSO cooperative communication assisted by DF relays based on error-correcting codes, particularly quasicyclic LDPC codes, has not been analyzed in the literature. The main contribution of this article is to propose a cooperative RF/FSO scheme assisted by a DF relay based on errorcorrecting codes, in particular quasicyclic LDPC codes. We also used the LDPC codes with spatial coupling (SC) to compare them with the quasicyclic LDPC codes and see their importance in cooperative optical communication and hybrid RF/FSO communication. We focus on the use of cooperative systems and error-correcting codes, particularly the quasicyclic LDPC codes with the association of a Layered BP (Belief Propagation) decoder at the relay level. We also used PPM modulation to modulate the data, the turbulent gamma-gamma air channel model, and AWGN to characterize the RF/FSO transmission channels and a maximum ratio combination technique (MRC) used at the receiver.

1.1. Motivation. Radiofrequency (RF) communication systems have advantages such as low power with a good operating range, and next to these, here, the line of sight (LOS) is not mandatory for communication. However, the problem is a lack of licensed spectrum with insufficient and also expensive data rates. On the contrary, free space optical (FSO) communication system have obtained the attention of convincing research due to its supremacy in the unlicensed optical spectrum, high bandwidth, high capacity, robustness to electromagnetic interference (EM), easy installation, and high data rate. Despite the benefits of FSO communication, there are limiting factors that play a major role. In general, over a distance of $1 \mathrm{~km}$, there is a significant deterioration in the quality of the signal represented in the FSO communication system, so communication with either the source (S) or the destination (D) is practically impossible. However, the FSO link is very sensitive to atmospheric effects such as pressure and temperature, which cause atmospheric turbulence along the propagation path.

However, an RF link is not affected by fog but is affected by absorption of $o_{2}$ and rain. Thus, RF and FSO 
have complementary characteristics. From this perspective, the combination of RF and FSO is seen as a powerful candidate for reliable communication at high data rates for wireless link networks. We can then note the fact that light rays pass poorly through matter. They are therefore generally confined to their environment. This is an advantage which improves information security (interception of data impossible from the outside), but which obviously limits the coverage and also makes this technology very sensitive to blocking phenomena.

It is in this launch that we are proposing an RF/FSO architecture in which we use RF links over long distances, that is to say between the aggregation node to the BST antennas, from a BTS antenna to the other BST antenna and FSO transmission over short distances of less than $1 \mathrm{~km}$ is from the BTS antenna towards the places of destination (inside cities). The proposed architecture reduces the cost of the system in both cases, increasing the reliability, security and performance of the network. FSO technology is likely to continue to be favored for short-range, low-cost broadband communications, and interference immunity is required. On the other hand, radio communication (RF) technology will continue to be used for longdistance transmission, or for communication systems operating in environments where weather conditions are of considerable impact. This architecture can be used in future generations (see Figure 1).

This document is organized as follows: in Section 2, the preliminaries, the system model, and the study model are introduced. Layered BP decoding is discussed in Section 3. Analysis of the simulation results is provided in Section 4. Finally, conclusions are drawn in Section 5.

\section{Model System and Channel Model}

\subsection{Model System}

2.1.1. Ratings. First, in what follows, we use letters, such as $H$, to denote a single parity matrix. We use $G$ to designate the generating matrix of the code, and $I$ represents the identity matrix. We use the notation for $x_{1}^{N}$ to designate a vector of dimension $\mathrm{N}\left(\boldsymbol{x}_{1}, \boldsymbol{x}_{2} \cdots \boldsymbol{x}_{N}\right)$.

2.1.2. The Quasicyclic LDPC Codes. Generally, a binary LDPC code is a linear block code specified by a particularly hollow binary parity check matrix: $H \boldsymbol{x}^{T}=\mathbf{0} \boldsymbol{o u} \boldsymbol{x}$ is a code word and $H$ can be considered in the form of a bipartite graph. Each column and row of $H$ represent a variable node and a control node, respectively. QC-LDPC codes are a very important class of LDPC codes.

There are different algebraic and combinatorial methods for constructing structured LDPC codes. The large circumference of the design of QC-LDPC code based on permutation matrices is of particular interest because of several advantages. The structure based on permutation matrices leads to an efficient implementation. It also provides a large minimum distance, which gives good cascade performance, since the minimum distance is exponentially proportional to the circumference. A large circumference QC-LDPC code with column weight $J$ and row weight $L$ can be represented by a parity check matrix $H$ :

$$
\mathrm{H}=\left[\begin{array}{cccc}
\mathrm{I}\left(p_{0,0}\right) & \mathrm{I}\left(p_{0,1}\right) & \cdots & \mathrm{I}\left(p_{0, L-1}\right) \\
\mathrm{I}\left(p_{1,0}\right) & \mathrm{I}\left(p_{1,1}\right) & \cdots & \mathrm{I}\left(p_{1, L-1}\right) \\
\vdots & \vdots & \ddots & \vdots \\
\mathrm{I}\left(p_{J-1,0}\right) & \mathrm{I}\left(p_{J-1,1}\right) & \cdots & \mathrm{I}\left(p_{J-1, L-1}\right)
\end{array}\right]
$$

First, where for $\mathbf{0} \leq \boldsymbol{j} \leq \boldsymbol{J}-\mathbf{1}, \mathbf{0} \leq \boldsymbol{l} \leq \boldsymbol{L}-\mathbf{1}, \boldsymbol{I}\left(\boldsymbol{p}_{\boldsymbol{j}, \boldsymbol{l}}\right)$ is a $p \times p$ circulating permutation matrix (CPM) formed by cyclically shifting each line by one identity matrix $\boldsymbol{p} \times \boldsymbol{p}$ I towards the right position $\boldsymbol{p}_{\boldsymbol{j}, l}$. It is obvious that $\mathrm{I}(0)$ is an identity matrix I of size $\boldsymbol{p} \times \boldsymbol{p}$. The value of $p$ is called the lift size (or degree of lift), and $\boldsymbol{p}_{\boldsymbol{j}, l}$ is called the CPM shift value I $\boldsymbol{I}\left(\boldsymbol{p}_{\boldsymbol{j}, l}\right)$. The parity check matrix $\mathrm{H}$ in (1) can be uniquely determined by the offset values. These offset values form a nonnegative matrix

$$
\mathrm{P}=\left[\begin{array}{cccc}
p_{0,0} & p_{0,1} & \cdots & p_{0, L-1} \\
p_{1,0} & p_{1,1} & \cdots & p_{1, L-1} \\
\vdots & \vdots & \ddots & \vdots \\
p_{J-1,0} & p_{J-1,1} & \cdots & p_{J-1, L-1}
\end{array}\right]
$$

This matrix $P$ is called the exponent matrix. In 2001, RM Tanner proposed a class of QC-LDPC (J; L)-regular codes [22], called Tanner (J; L) QC-LDPC codes which can be defined as follows: let $p$ be a premium and $F_{p}$ a first field. The general form of a parity check matrix of a Tanner code (J; L) QC-LDPC is represented by $H$ in (1) $p_{j, l}=b^{j} a^{l}$, for $0 \leq j \leq J-1,0 \leq j \leq J-1$, where $a$ and $b$ are nonzero integers with the orders $\mathrm{L}$ and $\mathrm{J}$ in the prime field $F_{p}$, respectively. Let $\alpha$ be a primitive root $(\mathrm{JL})$ th unit in $F_{p}$ where $J$ and $L$ are two positive integers and $L<J \leq 2$. So the offset values can be expressed as

$$
p_{j, l}=\alpha^{j \cdot L+l . J}(\bmod p)
$$

where $0 \leq j \leq J-1$ and $0 \leq j \leq J-1$.

Let us consider a prime field F7. It is clear that its sixth primitive unit roots, that is to say the primitive elements, are 3 and 5. Let $\alpha=3$. According to the definition above, the exponent matrix of the Tanner code $(2 ; 3)$ QC-LDPC of length 21 is

$$
\begin{aligned}
& P_{0}=\left[\begin{array}{lll}
3^{0.3+0.2} & 3^{0.3+1.2} & 3^{0.3+2.2} \\
3^{1.2+0.3} & 3^{1.3+1.2} & 3^{1.3+2.2}
\end{array}\right](\bmod 7) \text {, } \\
& P_{0}=\left[\begin{array}{lll}
1 & 2 & 4 \\
6 & 5 & 3
\end{array}\right](\bmod 7) \text {. }
\end{aligned}
$$




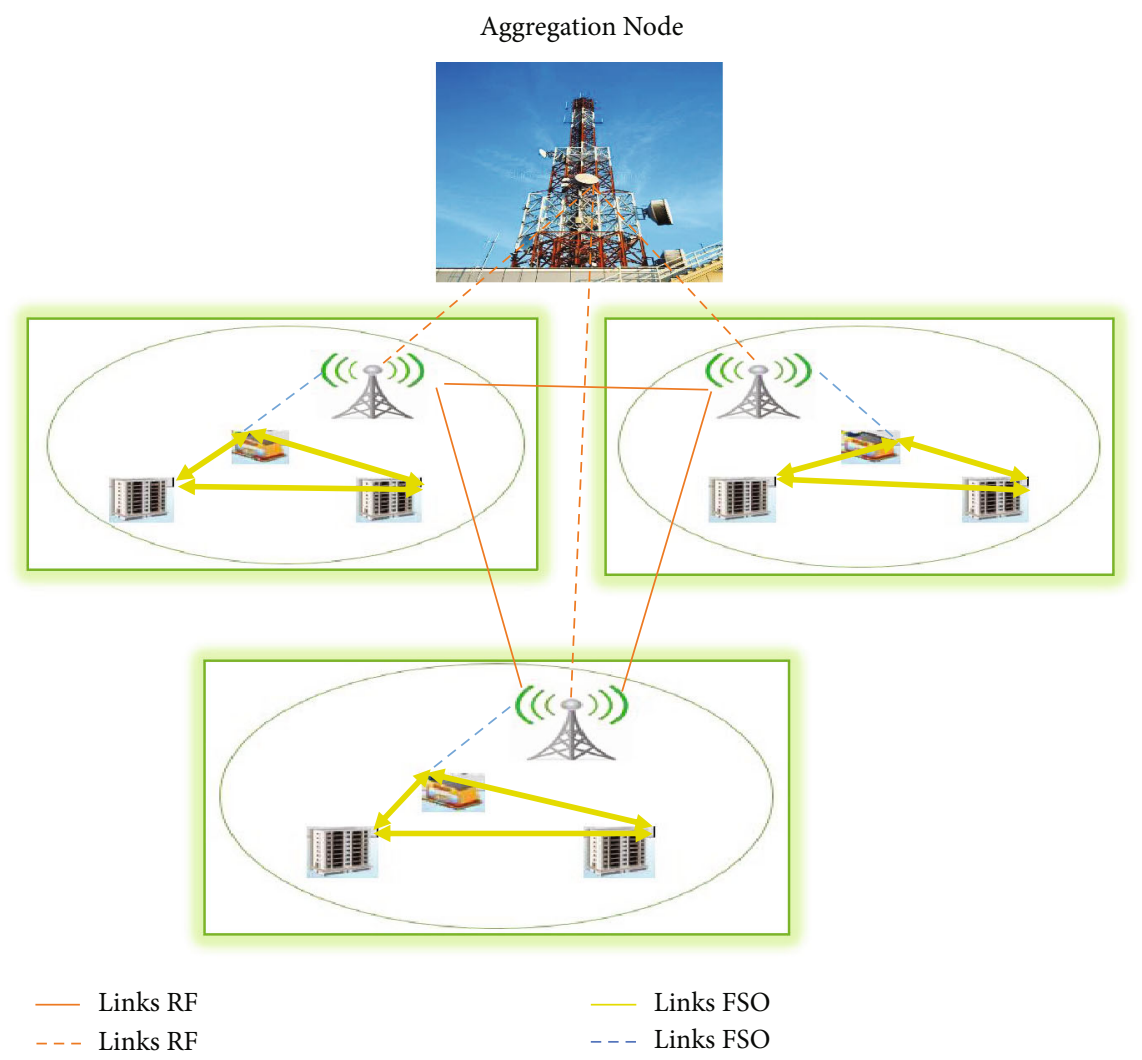

Figure 1: System architecture.

The parity check matrix of this Tanner QC-LDPC code $(2 ; 3)$ is denoted $H_{0}$. As the lifting size is 7 , the elements of the matrix $P_{0}$ represent $7 \times 7 \mathrm{CPM}$ in $H_{0}$. For example, item 1 in the first row and in the first column of $P_{0}$ represents the following $7 \times 7 \mathrm{CPM}$

$$
\mathrm{I}(1)=\left[\begin{array}{lllllll}
0 & 1 & 0 & 0 & 0 & 0 & 0 \\
0 & 0 & 1 & 0 & 0 & 0 & 0 \\
0 & 0 & 0 & 1 & 0 & 0 & 0 \\
0 & 0 & 0 & 0 & 1 & 0 & 0 \\
0 & 0 & 0 & 0 & 0 & 1 & 0 \\
0 & 0 & 0 & 0 & 0 & 0 & 1 \\
1 & 0 & 0 & 0 & 0 & 0 & 0
\end{array}\right]
$$

In $H_{0}$, the offset value of $I(1)$ is 1 .

2.1.3. The Space-Coupled (SC) LDPC Code. The construction method of our SC-LDPC code is a simple copy and shift operation. To make the implementation as easy as possible, we use the same basic QC-LDPC block code, supplied with the copy step [23]. The main idea of spatial code coupling is to introduce irregularity into regular codes. These higher column weight portions in an SC-LDPC code will benefit from the performance of the entire code word. The coupling process is based on subblocks. For example, if we set the cou- pling length to 5, with three $(3,15,1129)$ QC codes [23], the SC-LDPC code can be represented as

$$
H_{S C}=\left[\begin{array}{ccccccccccc}
I\left(p_{0,0}\right) & \cdots & I\left(p_{0,10}\right) & \cdots & I\left(p_{0,14}\right) & & & & & & \\
I\left(p_{1,0}\right) & \cdots & I\left(p_{1,10}\right) & \cdots & I\left(p_{1,14}\right) & & & & & & \\
I\left(p_{2,0}\right) & \cdots & I\left(p_{2,10}\right) & \cdots & I\left(p_{2,14}\right) & & & & & & \\
& & I\left(p_{0,0}\right) & \cdots & I\left(p_{0,4}\right) & \cdots & I\left(p_{0,10}\right) & \cdots & I\left(p_{0,14}\right) & & \\
& & I\left(p_{1,0}\right) & \cdots & I\left(p_{1,4}\right) & \cdots & I\left(p_{1,10}\right) & \cdots & I\left(p_{1,14}\right) & & \\
& & I\left(p_{2,0}\right) & \cdots & I\left(p_{2,4}\right) & \cdots & I\left(p_{2,10}\right) & \cdots & I\left(p_{2,14}\right) & & \\
& & & & & & I\left(p_{0,0}\right) & \cdots & I\left(p_{0,4}\right) & \cdots & I\left(p_{0,14}\right) \\
& & & & & & I\left(p_{1,0}\right) & \cdots & I\left(p_{1,4}\right) & \cdots & I\left(p_{1,14}\right) \\
& & & & & & I\left(p_{2,0}\right) & \cdots & I\left(p_{2,4}\right) & \cdots & I\left(p_{2,14}\right)
\end{array}\right] .
$$

The length of the code word for the SC-LDPC code will be $[c \times K-m \times(c-1)] \times b$, where $c$ represents the number of linked base codes and $m$ represents the coupling length.

2.1.4. Encoding of LDPC Codes. The LDPC codes have the particularity of being defined by their parity check matrix. As mentioned before, due to their complexity of encoding and decoding and the material means at the time, these codes had little impact on the coding theory community at the time of their discovery. Indeed, the trivial way to determine the code word is to use the generator matrix $G$, easily computable from the parity check matrix $H$. In the majority of cases, the generator matrix associated with a code whose matrix of parity check is low density. In the case where the code has no 
structure, the associated encoding complexity is important. To reduce this complexity, approaches that can be classified into two large families are considered.

The first consists in post processing the parity check matrix so as to introduce a form which is easy to encode. The second is based on the construction of a constrained parity check matrix, originally built to facilitate encoding. Regarding the first approach, Richardson and Urbanke in [24] propose a transformation of the parity check matrix by linear combinations of rows and columns into another parity check matrix of semitriangular shape the complexity of encoding then depends on a parameter characterizing the difference between the semitriangular matrix and the triangular matrix. Once the bits intervening in the semitriangular form obtained, the other redundancy bits are obtained by substitution. The second method consists in defining a constrained code structure. A first construction, very widely used, consists in constructing a parity check matrix defined by

$$
H=\left[H_{p} H_{s}\right] .
$$

The code word $x$ is then divided into an information word $c$ and a redundancy word $p$. The parity relation is then written:

$$
\begin{aligned}
H x^{t} & =0^{t}, \\
{\left[H_{p} H_{s}\right]\left[\begin{array}{c}
c^{t} \\
p^{t}
\end{array}\right] } & =0^{t}, \\
H_{p} p^{t} & =H_{s} c^{t} .
\end{aligned}
$$
by

We denote by $v^{t}$ propagation vector the vector defined

$$
v^{t}=H_{s} c^{t}
$$

It therefore follows that the set of parity bits can be deduced as follows:

$$
p^{t}=H_{p}^{-1} v^{t}
$$

This relation shows that the first constraint on the code is the existence of the inverse matrix $H_{p}^{-1}$. The matrix $H_{p}$ may be of triangular shape allowing, by simple substitution, the calculation of the redundancy bits. More particularly, the $H_{p}$ matrices of bidiagonal type are interesting for obtaining a simple encoding. The determination of the redundancy bits can be done by first calculating the projection vector then by an accumulation of this vector:

$$
p_{k}=p_{k-1}+v_{k}
$$

Another very interesting form of matrix of bidiagonal type is that illustrated in Figure 2. This type of structure has been retained within the framework of standardization.

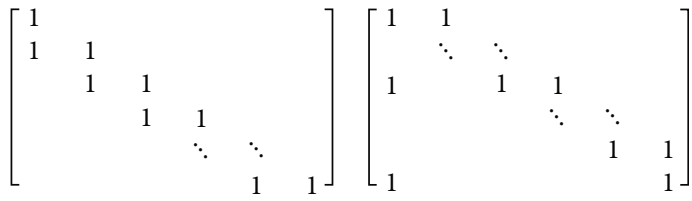

DVB-S2 IEEE 802.16e, 802.11n

(a)

(b)

Figure 2: Particular form of the matrix $H_{p}$ normalized in standards.

From what we have just seen, we can say that the encoding method is strongly linked to the construction of LDPC codes.

2.1.5. Study Model. We consider a cooperative double jump system without direct link using a DF relay with errorcorrecting codes, in particular the LDPC codes, where the source $\mathrm{S}$ transmits data to the destination $\mathrm{D}$ via a halfduplex relay R as shown in Figure 3. Here, a mixed RF-FSO link is developed in which the relay source transmission SR uses RF beams which is characterized by a Rayleigh fading distribution and the relay-destination transmission R-D uses an optical signal FSO by and is not the model lognormal. The transmitter uses BPSK modulation for signal broadcasting, the relay uses a PPM position modulation technique to transmit the optical signal to the destination, and the receiver uses the direct detection (DD) method to receive the optical sign.

2.2. Channel Model. In the following, each link is characterized in terms of respective signal-to-noise ratios. At the RF link, this link is established between $S$ and $\mathrm{R}$. At the source node, the modulated signal is noted $x_{1}^{N}$, where $N$ is the length of the coded signal. At the relay node, the signal received from the source and the signal retransmitted at the destination are noted $y_{1, S R}^{N}$ and $w_{1}^{N}$, respectively. The signal that is received by the $\mathrm{R}$ in phase $\mathrm{I}$ is given by

$$
y_{1, S R}^{N}=\sqrt{P_{S}} h_{1, S R}^{N} x_{1}^{N}+n_{1, S R}^{\mathrm{N}},
$$

where $x_{1}^{N}$ is the message signal transmitted from the source, $P_{s}$ is the transmit power of the source, and $n_{1, S R}^{N}$ is the additive white Gaussian noise of the channel (AWGN) S-R where $\sigma_{S R}^{2}=N_{01}$ is a unilateral power spectral density which is added at the relay and $h_{1, S R}^{N}$ represents the parameters of the channel between the source and the relay.

At the FSO link, this link is established between relay $\mathrm{R}$ and destination $\mathrm{D}$. Here, the relay retransmits the signal and $w_{1}^{N}$ in phase II, which encounters the induced atmospheric discoloration. The signal received at $\mathrm{D}$ by

$$
y_{1, R D}^{N}=\sqrt{P_{R}} h_{1, R D}^{N} w_{1}^{N}+n_{1, R D}^{N},
$$

where $w_{1}^{N}$ is the signal retransmitted from the relay, $P_{R}$ is the transmit power of the relay, and $n_{1, R D}^{N}$ is represented the additive noise to the receiver which consists of thermal noise, dark noise, and radiation background. Shot noise is created due to background radiation and is dominant over other 


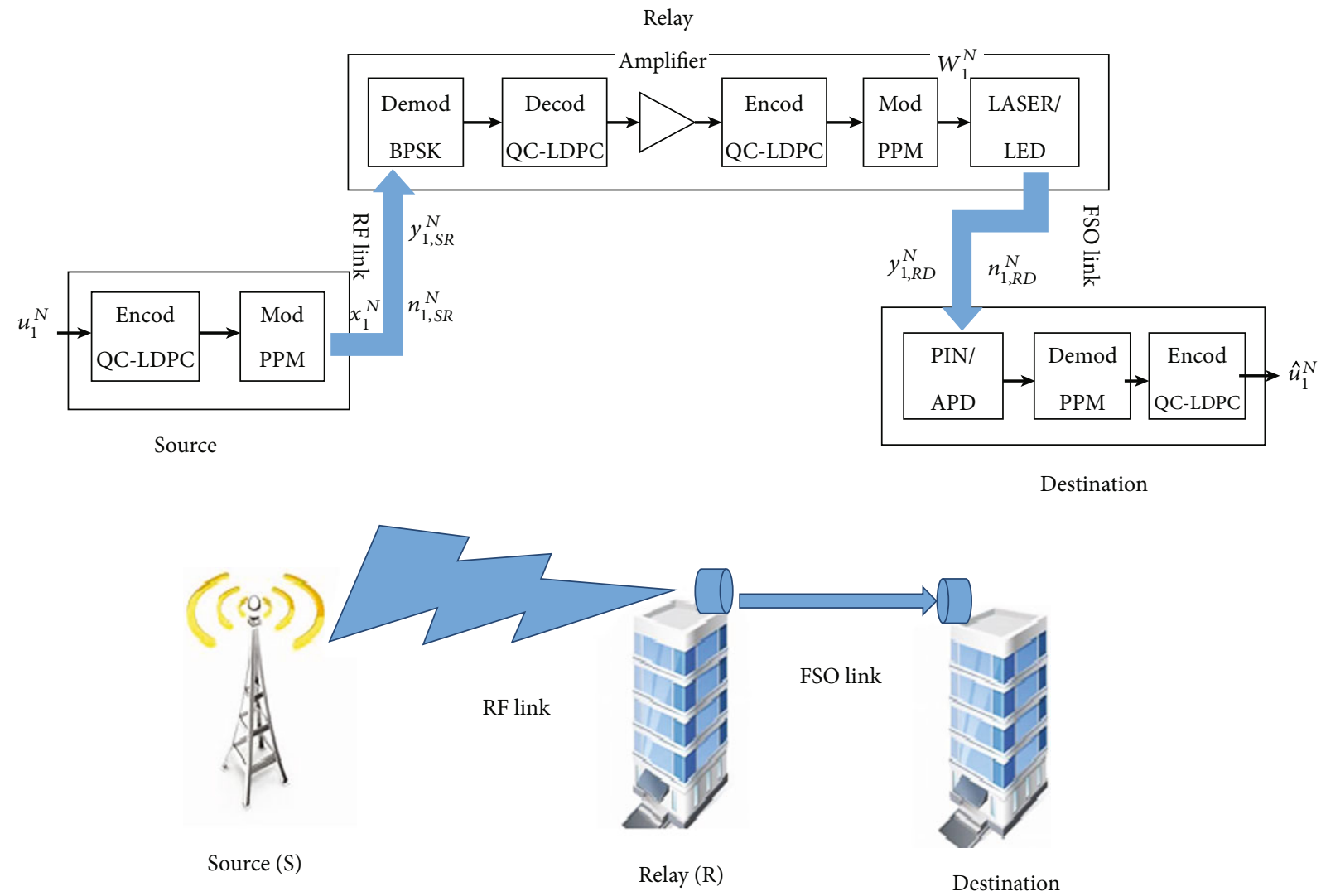

FIgURE 3: System model for DF based RF-FSO communication system.

noise components when the optical signal intensity is very high and can be modeled as AWGN with an average of zero and the variance $\sigma_{R D}^{2}=N_{02}$ is greater than 1 in the case of the gamma-gamma model and $h_{1, R D}^{N}$ represents the parameters of the channel between the relay and the destination. Throughout this part, we will assume that $P_{S}=P_{R}=1$.

With the parameters used in schema 3 , we proposed a second cooperative RF diagram (Figure 4) and wireless optics to increase the coverage of the system. We used parallel relays by an indirect path from the source to the destination. Source encodes bits using the quasicyclic LDPC codes, which is modulated using PPM modulation and sends by broadcast to the relays. The signals received by the relays are given as follows:

$$
\begin{aligned}
& y_{1, S R_{1}}^{N}=\sqrt{P_{S}} h_{1, S R_{1}}^{N} x_{1}^{N}+n_{1, S R_{1}}^{N}, \\
& y_{2, S R_{2}}^{N}=\sqrt{P_{S}} h_{2, S R_{2}}^{N} x_{1}^{N}+n_{2, S R_{2}}^{N}, \\
& y_{3, S R_{3}}^{N}=\sqrt{P_{S}} h_{3, S R_{3}}^{N} x_{1}^{N}+n_{3, S R_{3}}^{N} .
\end{aligned}
$$

At each relay, after converting the optical signal into an electrical signal, the latter will be demodulated using PPM demodulation and then decoded by an LDPC decoder using the Layered BP (Belief Propagation) algorithm.

The principle of decoding will be described in Section 3 . After these decoding operations, the signal is then reencoded as at the source. At the relay level, the electrical signal is converted into an optical signal and sent to the destination. Receipts by the destination are given as follows:

$$
\begin{aligned}
& y_{1, R_{1} D}^{N}=\sqrt{P_{R}} h_{1, R_{1} D}^{N} w_{1}^{N}+n_{1, R_{1} D}^{N}, \\
& y_{2, R_{2} D}^{N}=\sqrt{P_{R}} h_{2, R_{2} D}^{N} w_{2}^{N}+n_{2, R_{2} D}^{N}, \\
& y_{3, R_{3} D}^{N}=\sqrt{P_{R}} h_{3, R_{3} D}^{N} w_{3}^{N}+n_{3, R_{3} D}^{N},
\end{aligned}
$$

where $w_{1}^{N}, w_{2}^{N}$, and $w_{3}^{N}$ are the signals retransmitted from the relays; $P_{R}$ is the transmit power of the relay; and $n_{1, R_{1} D}^{N}, n_{2, R_{2} D}^{N}$, and $n_{3, R_{3} D}^{N}$ are represented the additive noise to the receiver which consists of thermal noise, dark noise, and background radiation; and $h_{1, R_{1} D}^{N}, h_{1, R_{2} D}^{N}$, and $h_{1, R_{3} D}^{N}$ represent the parameters of the channel between the relays and the destination. Throughout this part, we will assume that $P_{S}=P_{R}=1$.

2.2.1. Our Decode and Forward Scheme Based on Quasicyclic LDPC Codes. The decode and forward or DF is today one of the best cooperation techniques implemented at relay level in half-duplex mode when the state of the source-relay channel is better than that of the source-destination channel $\left(\mathrm{SNR}_{S-R}>\mathrm{SNR}_{S-D}\right)$.

In our model, we denote by $M$ the message to be sent of length $N$ (the length of the QC-LDPC code word and 


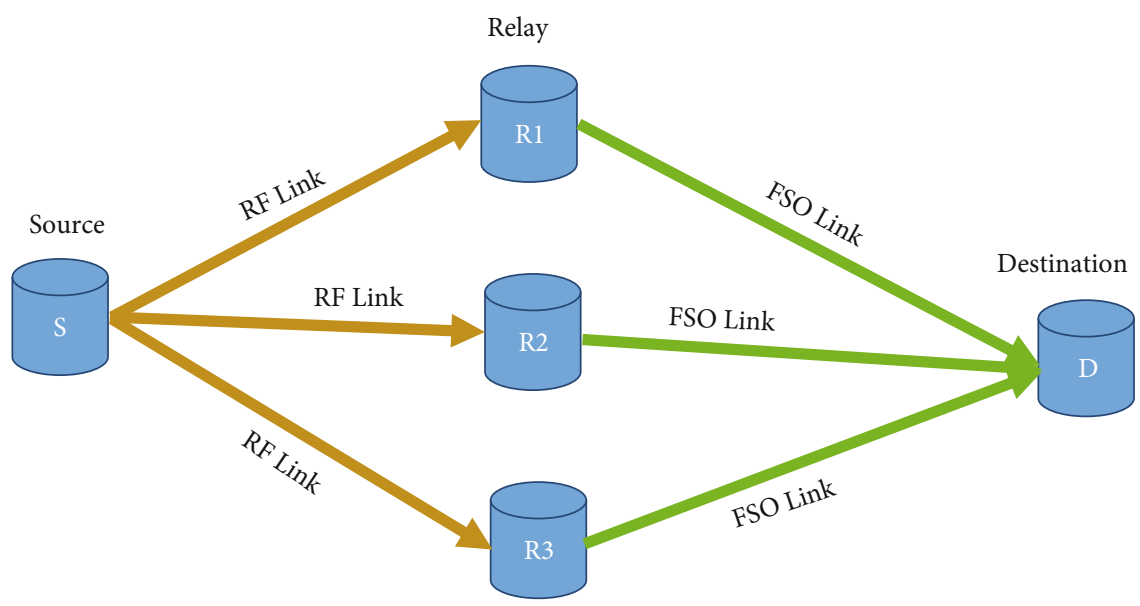

RF Link

FSO Link

FIGURE 4: Three relay-assisted cooperative RF/FSO system using QC-LDPC.

$N=1086)$ with $N=2^{n}(n \geq 0)$ composed of $K$ information bits and $(N-K)$ frozen bits. Let $\widehat{M}$ be the estimate of $M$. The quasicyclic LDPC coding of the $K$ information bits $(K=543)$ is carried out at the source.

(1) Source. The source encodes the information bits and the frozen bits using the quasicyclic LDPC codes according to the principle of polarization of the channel in $x_{1}^{N}$ containing $\mathrm{K}$ information bits and $N-K$ frozen bits. This signal $x_{1}^{N}$ modulated by a BPSK (Binary Phase Shift Keying) modulator is then sent to the relay using the AWGN channel of the RF link for the 1st time slot. The distance between the source and the destination is $10 \mathrm{~km}$ and is denoted $d$, and the distance between the source and the relay is $9.5 \mathrm{~km}$ and is denoted $l$ as shown in Table 1.

(2) Relay. At the relay level after converting the signal from the RF link into an electrical signal, the latter will be demodulated using BPSK demodulation and then decoded by a quasicyclic LDPC decoder using the Layered BP algorithm. The principle of decoding will be described in the following section.

After these decoding operations, the signal is then reencoded using the quasicyclic LDPC codes, this encoded signal will be modulated by a PPM (Pulse Position Modulation) modulator. This electrical signal is converted into an optical signal and sent to the destination using the gamma-gamma model of the FSO link at the 2nd time slot. We use $\lambda=1550 \mathrm{~nm}$ as the wavelength in $\mathrm{nm}$ which is related with the $\sigma_{R D}^{2}$ parameters of the gamma-gamma channel.

(3) Destination. The destination receives the signals $y_{1, R_{1} D}^{N}$, $y_{2, R_{2} D}^{N}$, and $y_{3, R_{3} D}^{N}$ from the FSO link and combines them by the MRC method (Maximum Ratio Combining) before demodulation with a PPM demodulator then decoding with quasicyclic LDPC codes to give the vector of estimated resulting symbols. Combination techniques are used to improve
TABLE 1: Simulation parameters.

\begin{tabular}{lcc}
\hline Parameter & Symbol & Value \\
\hline Wavelength & $\lambda$ & $1550 \mathrm{~nm}$ \\
Distance between source and destination & $d$ & $10 \mathrm{~km}$ \\
Distance between source and relay & $l$ & $9.5 \mathrm{~km}$ \\
Length of the QC-LDPC code word & $N$ & 1086 \\
Information bits & $K$ & 543 \\
Frozen bits & $N-K$ & 543 \\
Variance $\sigma_{R D}^{2}=N_{02}$ is greater than 1 in the & $\sigma_{R D}^{2}$ & $>1$ \\
case of the gamma-gamma model & $P_{S}$ & 1 \\
Transmit power of the source & $P_{R}$ & 1 \\
Transmit power of the relay & &
\end{tabular}

PPM (Pulse Position Modulation) modulator

BPSK (Binary Phase Shift Keying) modulator

The decoding results are compared to the source sequence and calculate the BER (Bit Error Rate) which is the number of error bits divided by the total number of bits transmitted.

the performance of the FSO link. Diversity reception systems use linear combination techniques such as equal gain combination (EGC), selection combination (SC), and maximum ratio combination (MRC). The decoding results are compared to the source sequence and calculate the BER (Bit Error Rate) which is the number of error bits divided by the total number of bits transmitted.

\section{Algorithm Layered BP}

The Layered BP (Belief Propagation) algorithm is the direct application of shuffle-type scheduling introduced in [25]. This scheduling is also known under the name of Horizontal shuffle, where still Turbo Like message passing algorithm [26].

This sequencing consists in considering the LDPC code as a parallel concatenation of $M$ parity codes. The code is 


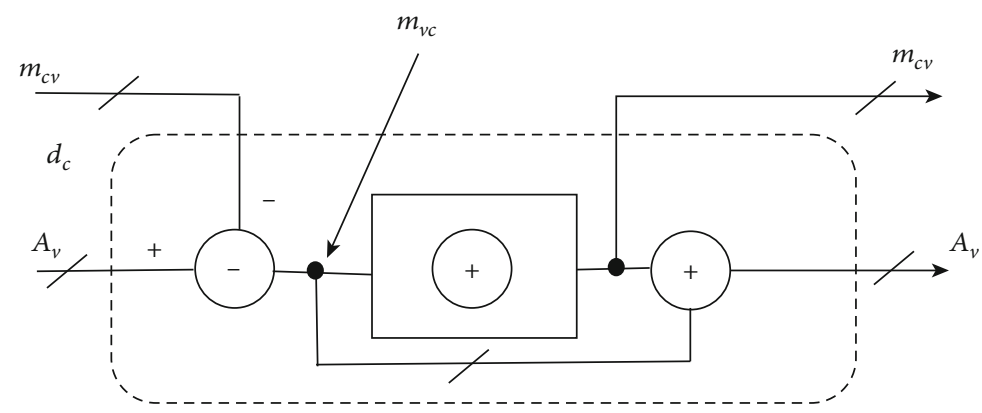

FIgURE 5: Form Diagram of the decoder associated with a decoding of a parity equation in a Layered BP sequencing. $d_{c}$ Represents the number of bits involved in the parity equation.

decoded like a parallel Turbo code: the $M$ parity codes are decoded one after the other. Unlike conventional scheduling where a node is updated once per iteration, a node connected to $d_{v}$ parity equations will be updated $d_{v}$ times during the same iteration. The principle of this sequencing is therefore always using the latest updated information. This decoding strategy explains the increase in the convergence speed of the decoding algorithm compared to the BP algorithm with flood sequencing.

The main idea of belief propagation- (BP-) based algorithms is to treat the symbols received iteratively in concatenated steps which can be seen on the Tanner graph as a horizontal step followed by a vertical step to improve reliability. Of each decoded code symbol, reliability measures calculate code symbols at the end of all decoding iterations that are used as inputs for the next iteration. This decoding iteration algorithm continues until a certain stop criterion is satisfied. To reduce the complexity of the decoding during the implementation of the decoder, we propose a Layered BP algorithm. This decoding algorithm can be described in three stages. A first step consists in calculating the messages $m_{v c}$ propagating from the nodes of variable $v$ to the node of parity $c$ for each layer considered:

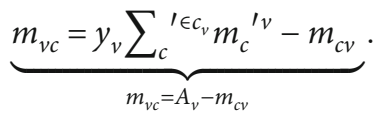

We recall $A_{v}$ which is the posterior information associated with node $v$. Unlike the decoding equations presented above, the posterior information is updated several times in the same iteration.

From the $m_{v c}$ messages corresponding to the sum of the observation of the channel and the a priori information, the parity equation is checked. The resolution of the parity equation allows the generation of extrinsic information $m_{c v}$ associated with each element involved in the parity equation. The last step therefore consists in updating the information a posteriori associated with each node $v$ :

$$
\underbrace{A_{v}=y_{v}+y_{v}+\sum_{c}{ }^{\prime} \epsilon c_{v / c} m_{c}{ }^{\prime v}+m_{c v}}_{A_{v}=m_{v c}+m_{c v}} .
$$

These two decoding equations allow us to consider the decoder scheme illustrated in Figure 5. The writing of the decoding algorithm highlights two important points. First, the decoder will have to work with two memories. The first, of depth $N$, stores the posterior information $A_{v}$, while the second stores the extrinsic information associated with each node involved in a parity equation. The number of elements to memorize is therefore equal to the number of nonzero elements in the parity check matrix. This achievement illustrates the importance of suboptimal decoding algorithms that reduce the number of different messages generated by a control node.

Thus, the layered algorithm and scheduling are particularly well suited to the structure studied and more generally to codes whose parity check matrices are constructed from permuted identity matrices. This combination explains the interest aroused by this family of QC-LDPC codes in standardization groups and in the context of the hardware implementation of a decoder.

Constraints on the construction of the code: realization the use of a BP layered decoding algorithm is supposed to solve the parity equations in a sequential way. An element involved in a parity equation must not be treated at the same time by another parity equation. To be able to guarantee a certain operating rate, it is necessary that several parity equations are decoded in parallel. The parity check matrix must therefore be constrained so as to guarantee that the resolution of $p$ parity equations only involves a parity node once. This constraint results in the fact that $M / p$ submatrices constructed from $p$ lines decoded simultaneously have at most only one nonzero element per column. The factor of maximum parallelism must thus be lower than $\left\lfloor M d_{v}^{\max }\right\rfloor$ where $d_{v}^{\max }$ the maximum degree of the nodes of parities. A subset of codes respecting this constraint is the set of codes whose parity check matrices are constructed from permuted identity matrices of sizes $p \times p$. In the case of the structure studied, the permuted identity matrices are of size $z \times z$ thus allowing a degree of parallelism equal to $z$. This construction is therefore particularly suitable for a Layered $\mathrm{BP}$ type algorithm (the literal translation of which is BP per layer). A block diagram of the decoding process is illustrated in Figure 6.

\section{Simulation and Results}

The systems described above were simulated on Matlab. The purpose of these simulations is to study the 


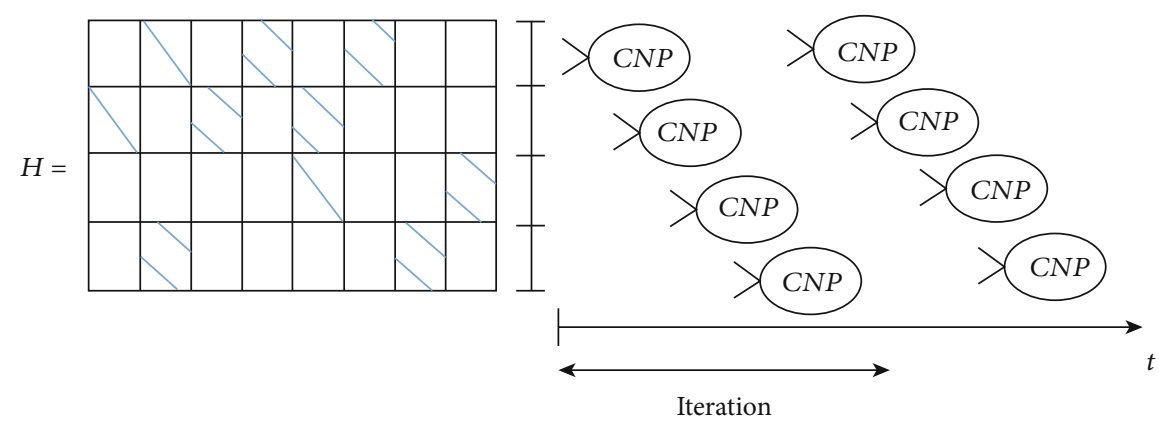

FIGURE 6: Diagram of the decoding by the Layered BP algorithm of a QC-LDPC code whose parity check matrix H is constructed from permuted identity matrix. The concept of layer decoding (Layer) is illustrated here.

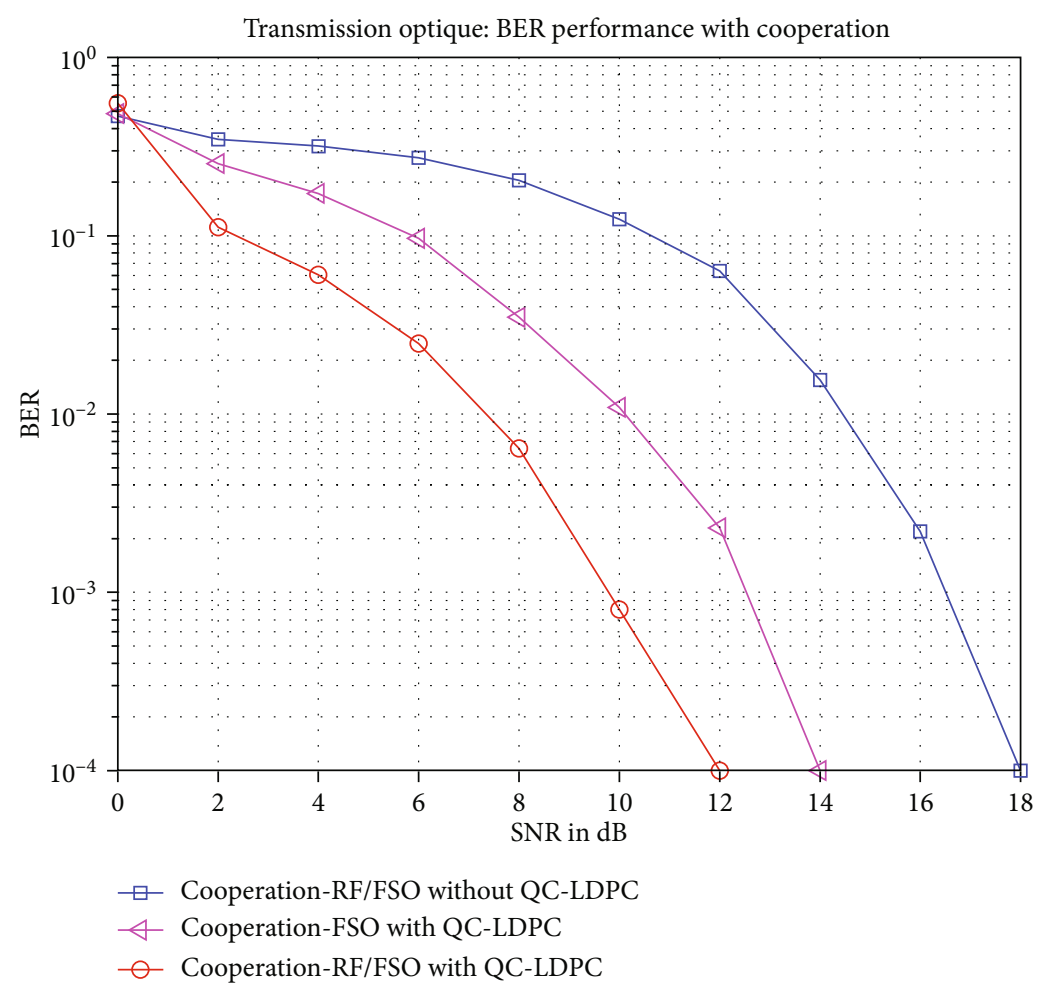

Figure 7: Comparison of three cooperative schemes using a DF relay.

performance of the RF/FSO system with spatial diversity in the context of PPM modulation and the use of errorcorrecting codes, in particular QC-LDPC codes. The effects of atmospheric turbulence have been modeled by the gamma-gamma distribution for high turbulence, the lognormal distribution for low turbulence for the first time slot, and additive Gaussian white noise (AWGN) for the second time slot.

By considering a gamma-gamma model FSO optical channel, an AWGN model RF channel, PPM modulation, and using QC-LDPC codes (Figure 3), Figure 7 presents the BER performances obtained as a function of SNR in decibel (dB) to compare the performances of the RF/FSO cooperative system using a QC-LDPC DF relay (Figure 3), a cooperative system using an RF/FSO relay without the QC-LDPC codes and a FSO cooperative system.
Figure 7 shows a summary and summary comparative study of the scenarios of an RF/FSO scheme using the QCLDPC codes set out above, a cooperative FSO scheme using the QC-LDPC FSO codes, and an RF/FSO scheme without the use of QC-LDPC codes. It can be seen there that the $\mathrm{RF} / \mathrm{FSO}$ transmission using the error-correcting codes, in particular, the QC-LDPC codes, presents better performance in the cases of FSO cooperation with QC-LDPC and cooperation without QC-LDPC. RF/FSO transmission with relay in decoding before retransmission (DF) mode using QC-LDPC codes seems to prevail over cooperative FSO transmission with the use of QC-LDPC codes and RF/FSO transmission without the use of QC codes-LDPC in terms of quality performance.

The simulations in Figures 8 and 9 allow us to appreciate in comparison to an RF/FSO transmission using DF parallel 


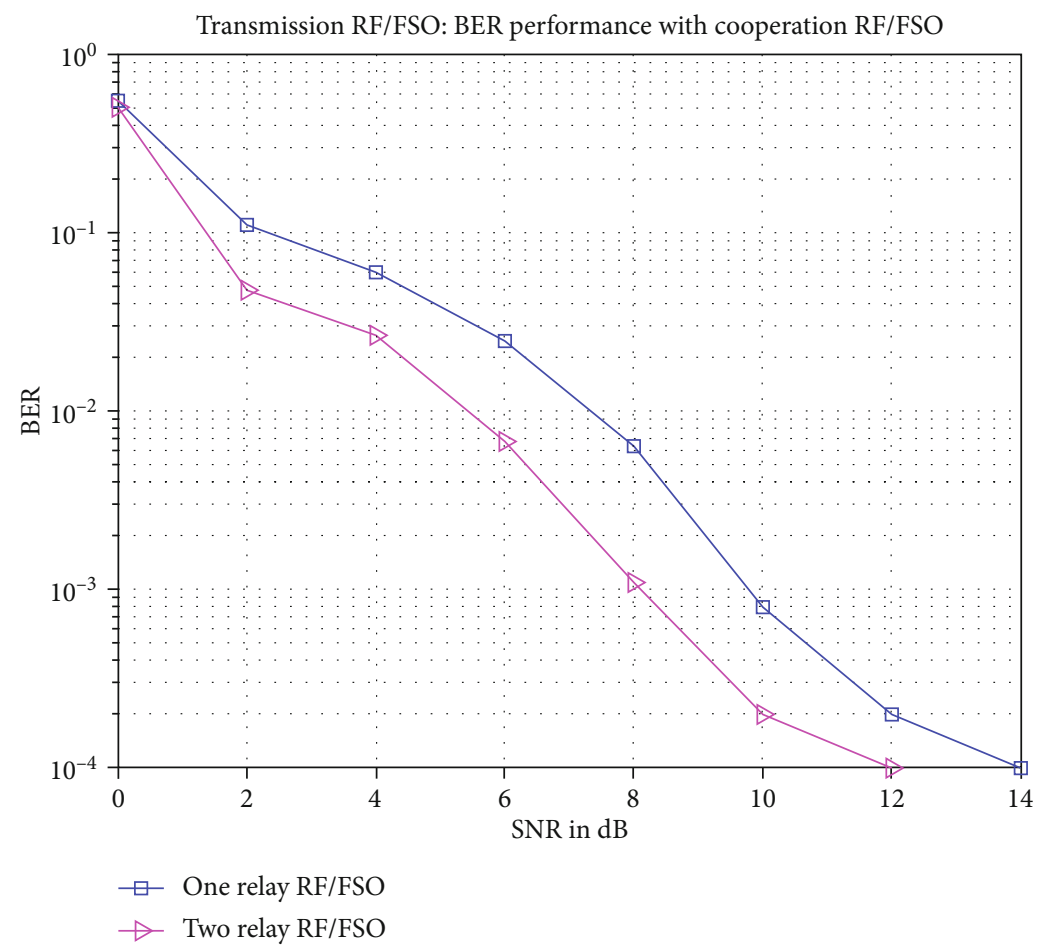

FIgURE 8: Strong turbulence with two DF RF/FSO relays.

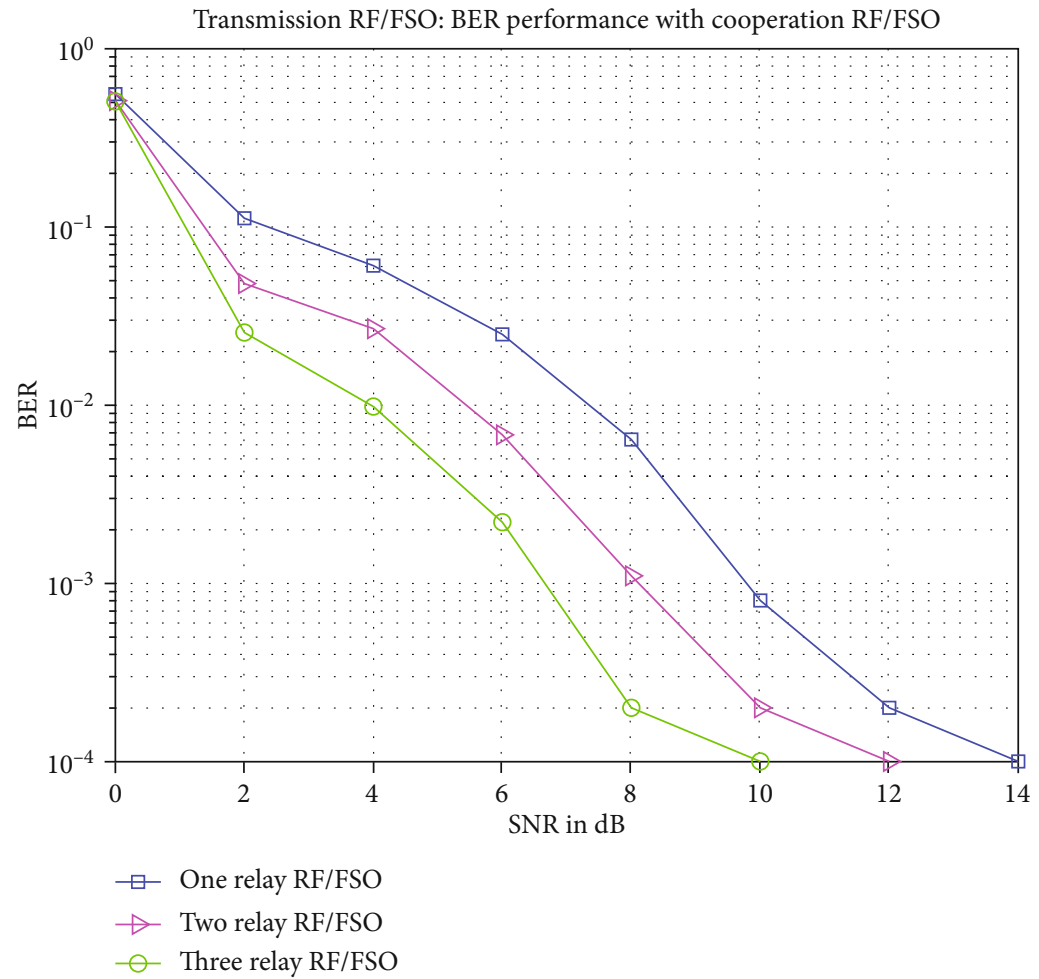

FIGURE 9: Strong turbulence with three relays DF RF/FSO (Figure 4).

relays using the QC-LDPC codes and the system stated above (Figure 3), the impact of using RF/FSO relays in the following cases: use of a DF relay (Figure 3), two parallel DF relays and three parallel DF relays (Figure 4). We used the gamma- gamma model, the PPM modulation, and the QC-LDPC codes on each diagram.

The graph in Figure 9 illustrates the performance comparison between an RF/FSO communication system using a 


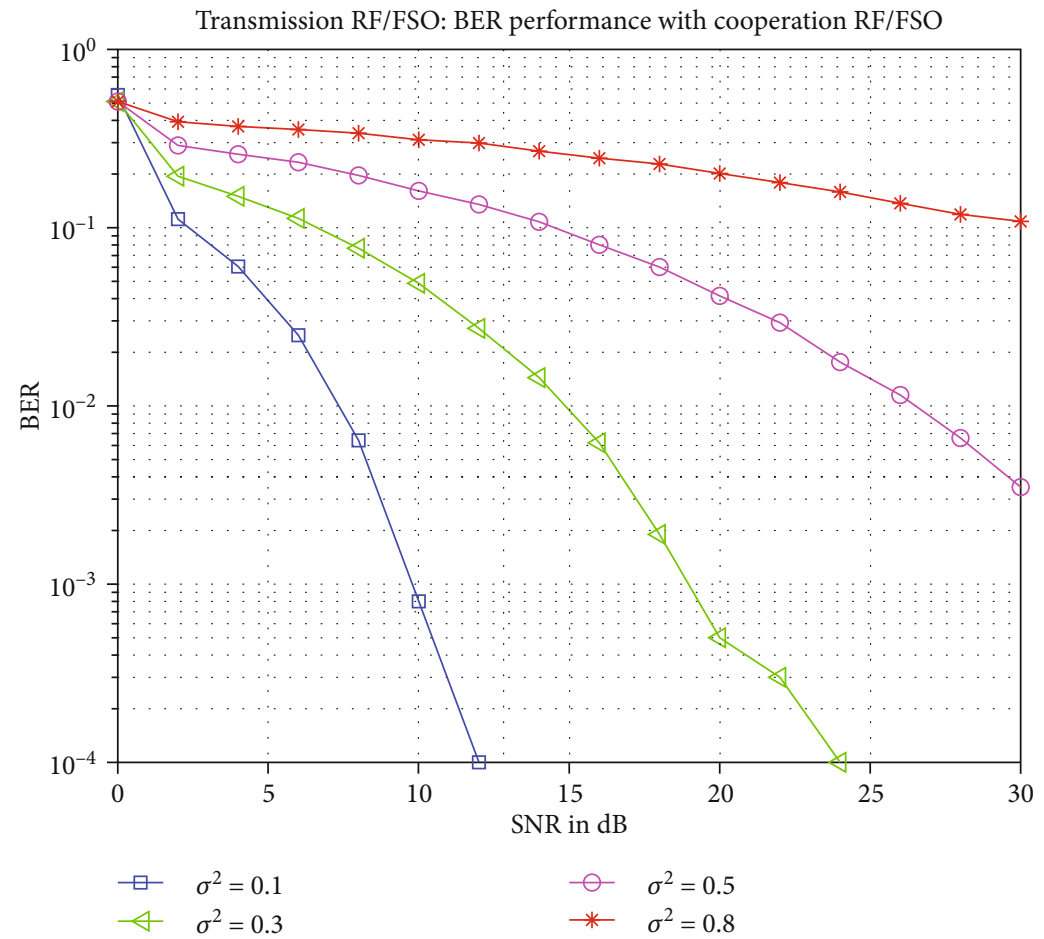

FIGURE 10: Cooperation RF/FSO using a DF QC-LDPC relay under several levels of turbulence.

DF relay and an RF/FSO communication system using two parallel relays when using PPM and QC-LDPC codes. With a BER (bit error rate) of $10^{-4}, \mathrm{RF} / \mathrm{FSO}$ with two parallel relays has a gain of $2 \mathrm{~dB}$ compared to an RF/FSO communication scheme.

Figure 9 shows the performance comparison between a mixed RF/FSO network using a DF relay, an RF/FSO communication system using two parallel relays, and a communication system using 3 relays when using PPM, BPSK, and QC-LDPC codes. With a BER (bit error rate) of $10^{-4}$, the $\mathrm{RF} / \mathrm{FSO}$ with 3 parallel relays has a gain of $2 \mathrm{~dB}$ compared to an $\mathrm{RF} / \mathrm{FSO}$ communication scheme with 2 relays and a gain of $4 \mathrm{~dB}$ compared to an RF/FSO communication scheme with a relay. We can see that RF/FSO systems using PPM, BPSK, and QC-LDPC with 3 relays have better overall performance compared to an RF/FSO system with 2 relays and 1 relay using PPM modulation and QC-LDPC codes in BER terms. By gradually adding a relay, the gain increases each time.

Considering RF/FSO communication using a relay of Log-normal model, AWGN, QC-LDPC codes, and using PPM modulation, Figure 10 presents the BER performances obtained as a function of SNR in decibel (dB) for several values of variance $\sigma^{2}$.

The parameter $\sigma^{2}$ is linked to the scintillation index. The latter is calculated from atmospheric conditions. As expected, in a more turbulent atmospheric condition which is due to an increase in the value of variance of normal log and AWGN, there is a degradation of the error rate. So the more we decrease the values $\sigma^{2}$, the more the performance of the $\mathrm{RF} / \mathrm{FSO}$ system increases.
Figure 11 shows the BER performances obtained as a function of SNR in decibel (dB) for all turbulent conditions (weak, moderate, and strong) using PPM, BPSK modulation, and QC-LDPC codes.

Figure 11 shows the performance comparison in a mixed $\mathrm{RF} / \mathrm{FSO}$ network using three parallel DF relays using the QCLDPC codes for all turbulent conditions (low, moderate, and strong). Here, we got BER performance better for weak and moderate turbulent regimes compared to strong. It can be seen that the performance of the links is better overall when the channel is slightly turbulent.

In Figure 12, simulations were performed on an RF/SFO transmission channel (AWGN, gamma-gamma) and modulations (PPM, BPSK) to see the impact of the variation in the performance of QC-LDPC codes in transmission RF/FSO using DF relays.

Figure 12 shows the comparison of performances obtained for an RF/FSO transmission using errorcorrecting codes, particularly QC-LDPC codes with different yields. With a BER (bit error rate) of $10^{-4}$, the RF/FSO transmission using QC-LDPC codes of efficiency $1 / 2$ presents a gain of $2 \mathrm{~dB}$ compared to an RF/FSO communication using 2/3 efficiency QC-LDPCs and a gain of $4 \mathrm{~dB}$ compared to an RF/FSO transmission using 4/5 efficiency QC-LDPCs. It can be seen that RF/FSO systems using QC-LDPC with low efficiency have better overall performance compared to an $\mathrm{RF} / \mathrm{FSO}$ system using QC-LDPC codes with high efficiency.

The graph in Figure 13 illustrates the performance comparison between a mixed RF/FSO communication system and mixed FSO/RF communication systems using QCLDPC codes but also using PPM and BPSK modulations. In 


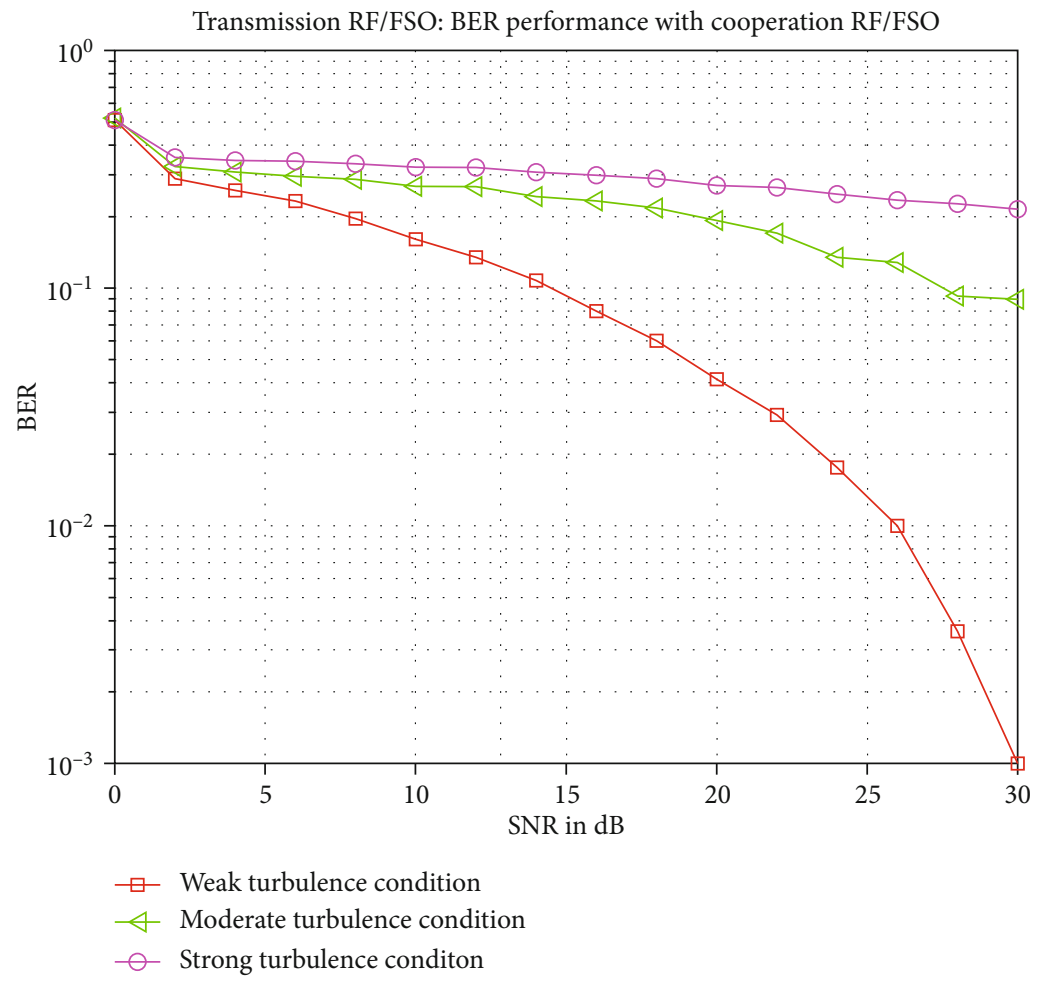

FIGURE 11: DF-based mixed RF-FSO system under all turbulence conditions.

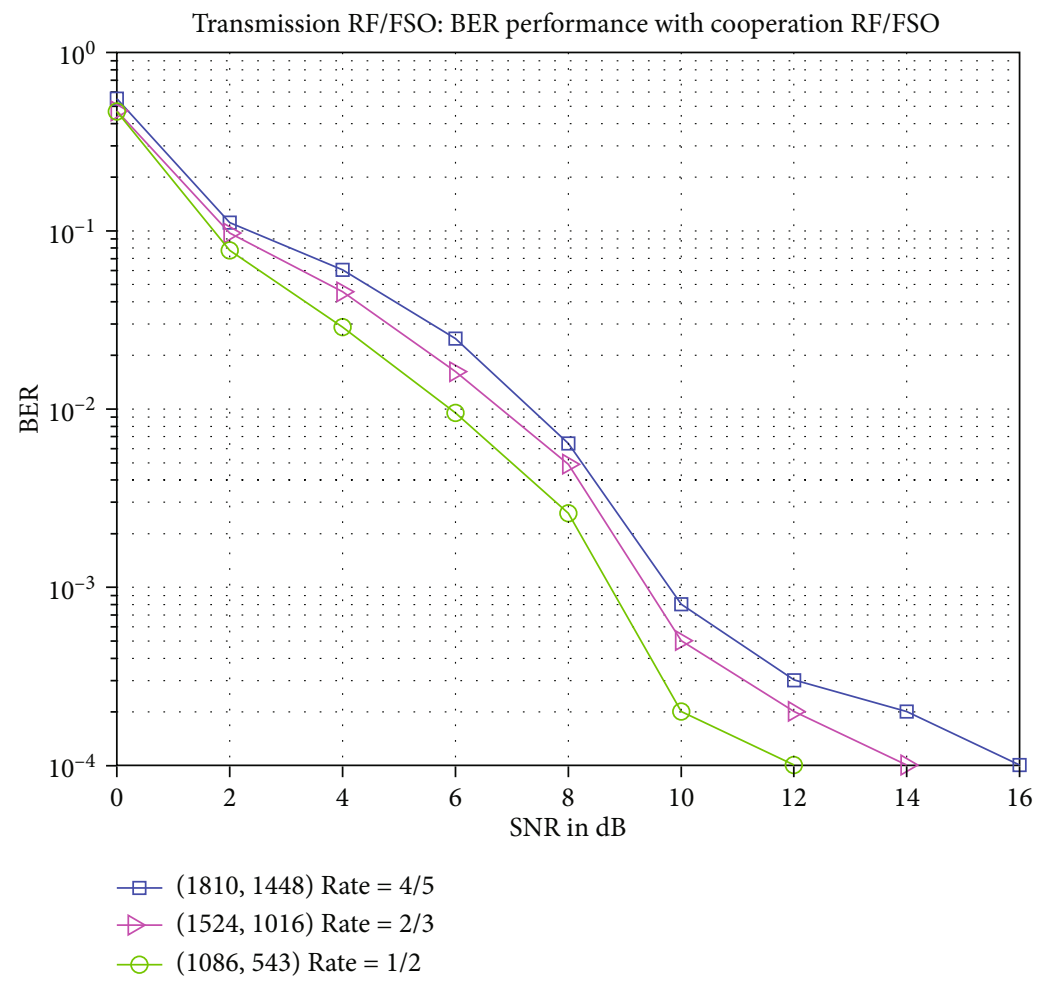

FIGURE 12: Performances obtained with QC-LDPC codes with returns 1/2, 2/3 and 4/5 for RF/FSO transmission.

this case, we used a relay in the middle for RF/FSO communication and FSO/RF communication.

The simulation in Figure 13 allows us to appreciate in comparison to an RF/FSO transmission using a DF relay in the middle and an FSO/RF transmission also using a DF relay in the middle with the distances. On both transmissions, we used the QC-LDPC codes. We can see for a $12 \mathrm{~dB}$ SNR us a BER (bit error rate) of $10^{-4}$ for $\mathrm{RF} / \mathrm{FSO}$ 


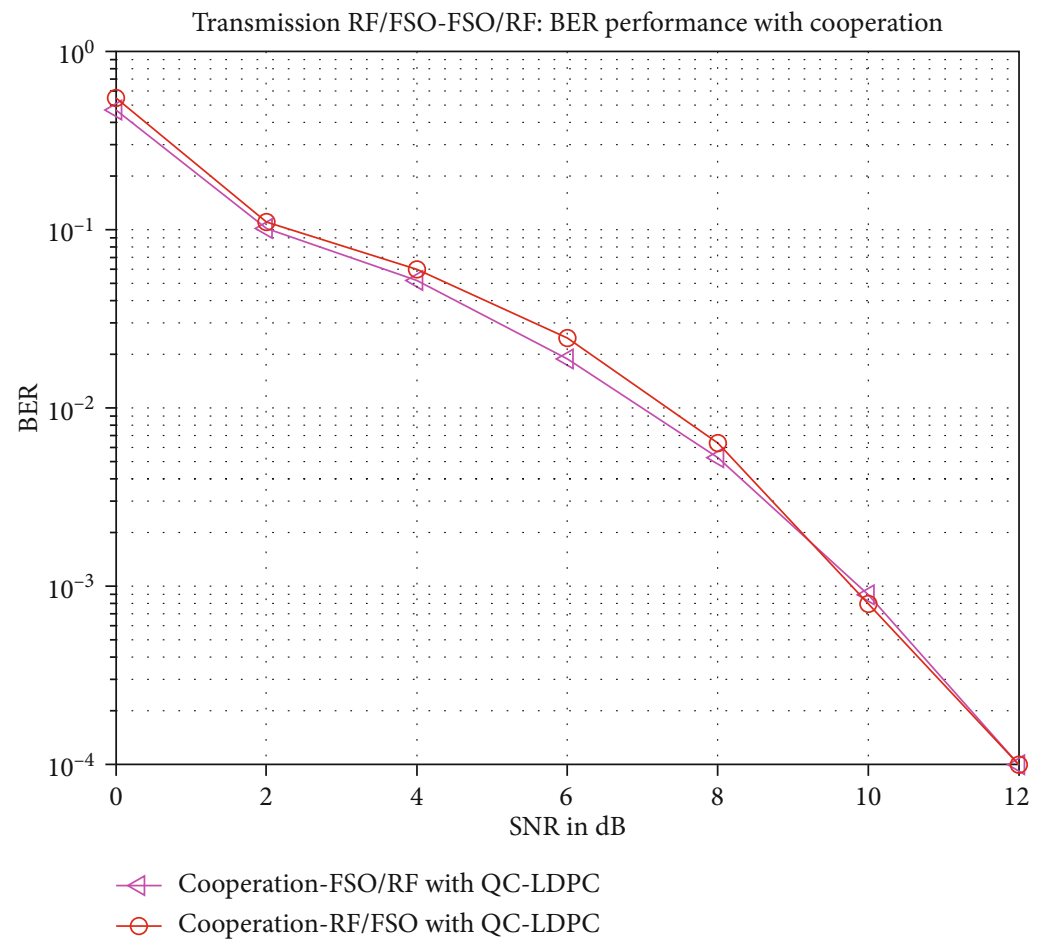

FIGURE 13: RF/FSO and FSO/RF transmission using QC-LDPC.

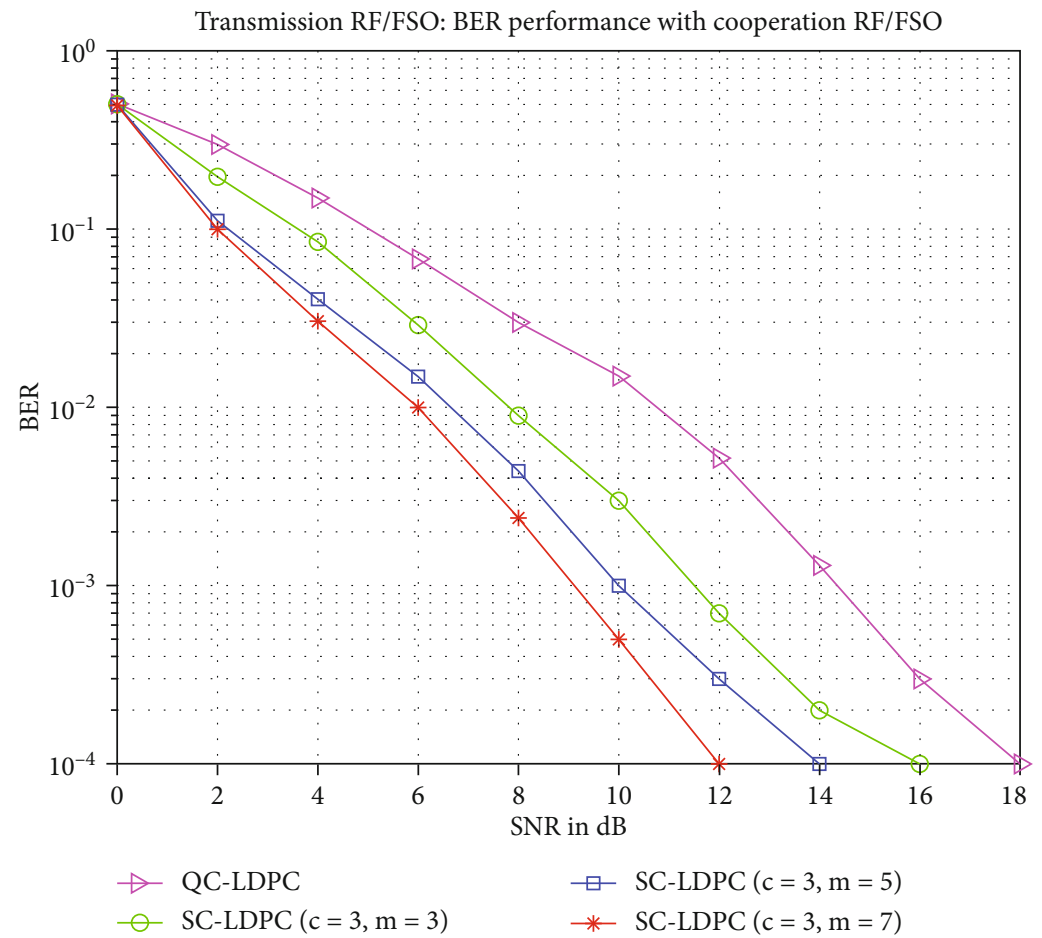

FIGURe 14: Performance of SC-LDPC codes compared to QC-LDPC codes in hybrid RF/FSO cooperative transmission.

transmission and FSO/RF transmission. We can then conclude that the RF/FSO and FSO/RF performance depends on the position of the relay, if we place the relay in the middle for both types of transmission we have the performance. This means that in relation to the needs of the users, the RF/FSO transmission or the FSO/RF transmission are used.

The BER performance as a function SNR [dB] of the LDPC codes in the cooperative and hybrid optical transmission RF/FSO are summarized in Figure 14. It should be noted 
that all the SC LDPC codes are derived from the same basic code QC-LDPC.

As we can see in the figure above, the spatial coupling of the basic code QC-LDPC provides a higher coding gain in mixed RF/FSO communication. Performance improves as the length of the coupling increases. The results showed that the SC-LDPC codes perform better than the basic LDPC QC code. From the implementation point of view, the structure of the SC-LDPC code is suitable for an optical communication application due to its low complexity.

\section{Conclusion}

In this article, the performance of an RF-FSO system using error-correcting codes including QC-LDPC codes and mixed transmission with $N$ relays RF/FSO also using QC-LDPC was evaluated. Using several simulation results, the performances of the mixed RF-FSO system considered using QC-LDPC under weak moderate and strong atmospheric conditions were evaluated. It is shown that hybrid RF/FSO systems give better performance in atmospheric conditions of low turbulence compared to atmospheric conditions of high turbulence. We have shown the impact of using QC-LDPC in $\mathrm{RF} / \mathrm{FSO}$ water communication. In terms of performance, systems using QC-LDPCs give better performance compared to systems without the error-correcting codes. We also presented an analysis on the performance of QC-LDPC. Finally, we also evaluated the performance of the RF/FSO and FSO/RF systems using a DF relay in the middle associated with the QC-LDPC at each relay. We also evaluated the performance of the SC-LDPC codes compared to the QC-LDPC codes in cooperative optical transmission and mixed RF/FSO.

In future work, we are interested in FSO/RF/FSO hybrids using error-correcting codes. In future work, researchers can replace the RF link by optical fiber to increase the start of transmission and the security of the system. We also plan to use GC-LDPC codes to increase performance in the case of cooperative optical transmission and to do an in-depth study of SC-LDPC codes in cooperative optical transmission. We are also considering using LDPC codes with deep learning in wireless optical communications. These simulations were performed in Figure 4 with the use of three relays to compare the QC-LDPC and SC-LDPC.

\section{Data Availability}

The interest of this article concerns the use of hybrid RF/FSO systems. We have used error coordinators like QC-LDPC codes and SC-LDPC codes in this system. We have compared them with conventional systems. In the architecture that you have proposed, the results show that we have the best performance as with conventional systems. This architecture could be used in future generations like $5 \mathrm{G}$.

\section{Conflicts of Interest}

The authors declare that they have no conflicts of interest.

\section{References}

[1] H. G. Sandalidis, T. A. Tsiftsis, G. K. Karagiannidis, and M. Uysal, "BER performance of FSO links over strong atmospheric turbulence channels with pointing errors," IEEE Communications Letters, vol. 12, no. 1, pp. 44-46, 2008.

[2] C. Abou-Rjeily, "Performance analysis of selective relaying in cooperative free-space optical systems," Journal of Lightwave Technology, vol. 31, no. 18, pp. 2965-2973, 2013.

[3] S. Song, Y. Liu, Q. Song, and L. Guo, "Relay selection and link scheduling in cooperative free-space optical backhauling of $5 \mathrm{G}$ small cells," in 2017 IEEE/CIC International Conference on Communications in China (ICCC), pp. 1-6, Qingdao, China, 2017.

[4] M. A. Kashani and M. Uysal, "Outage performance and diversity gain analysis of free-space optical multi-hop parallel relaying," Journal of Optical Communications and Networking, vol. 5, no. 8, pp. 901-909, 2013.

[5] I. Gueye, I. Dioum, K. W. Keita et al., "Performance of cooperative system based on LDPC codes in wireless optical communication," Proceedings of the Future Technologies Conference (FTC) 2019. FTC 2019, pp. 745-757, Springer.

[6] N. Varshney, A. K. Jagannatham, and P. K. Varshney, "Cognitive MIMO-RF/FSO cooperative relay communication with mobile nodes and imperfect channel state information," IEEE Transactions on Cognitive Communications and Networking, vol. 4, no. 3, pp. 544-555, 2018.

[7] Z. Jing, Z. Shang-hong, Z. Wei-hu, and C. Ke-fan, "Performance analysis for mixed FSO/RF Nakagami-m and exponentiated weibull dual-hop airborne systems," Optics Communications, vol. 392, pp. 294-299, 2017.

[8] B. Ashrafzadeh, E. Soleimani-Nasab, M. Kamandar, and M. Uysal, "A framework on the performance analysis of dual-hop mixed FSO-RF cooperative systems," IEEE Transactions on Communications, vol. 67, no. 7, pp. 4939-4954, 2019.

[9] S. Anees and M. R. Bhatnagar, "Performance evaluation of decode-and-forward dual-hop asymmetric radio frequencyfree space optical communication system," IET Optoelectronics, vol. 9, no. 5, pp. 232-240, 2015.

[10] N. Singhal, A. Bansal, and A. Kumar, "Performance evaluation of decode-and-forward-based asymmetric SIMO-RF/FSO system with misalignment errors," IET Communications, vol. 11, no. 14, pp. 2244-2252, 2017.

[11] S. Sharma, A. S. Madhukumar, and S. Ramabadran, "Switching based hybrid FSO/RF transmission for DF relaying system," in 2018 IEEE Wireless Communications and Networking Conference (WCNC), pp. 1-6, Barcelona, Spain, 2018.

[12] M. Torabi and N. Mohammadi, "Partial relay selection with feedback delay in an AF cooperative relaying RF-FSO system with spectrum sharing," in 2019 2nd West Asian Colloquium on Optical Wireless Communications (WACOWC), pp. 2630, Tehran, Iran, 2019.

[13] X.-Q. Jiang, H. Hai, H.-M. Wang, and M. H. Lee, "Constructing large girth QC protograph LDPC codes based on PSD-PEG algorithm," IEEE Access, vol. 5, pp. 13489-13500, 2017.

[14] X.-Y. Hu, E. Eleftheriou, and D. M. Arnold, "Regular and irregular progressive edge-growth Tanner graphs," IEEE Transactions on Information Theory, vol. 51, no. 1, pp. 386398, 2005.

[15] C. Chen, B. Bai, and X. Wang, "Construction of nonbinary quasi-cyclic LDPC cycle codes based on singer perfect 
difference set," IEEE Communications Letters, vol. 14, no. 2, pp. 181-183, 2010.

[16] J. Li, K. Liu, S. Lin, and K. Abdel-Ghaffar, "Algebraic quasicyclic LDPC codes: construction, low error-floor, large girth and a reduced-complexity decoding scheme," IEEE Transactions on Communications, vol. 62, no. 8, pp. 2626-2637, 2014.

[17] C. Chen, B. Bai, G. Shi, X. Wang, and X. Jiao, "Nonbinary LDPC codes on cages: structural property and code optimization," IEEE Transactions on Communications, vol. 63, no. 2, pp. 364-375, 2015.

[18] Q. Diao, J. Li, S. Lin, and I. F. Blake, "New classes of partial geometries and their associated LDPC codes," IEEE Transactions on Information Theory, vol. 62, no. 6, pp. 2947-2965, 2016.

[19] M. P. C. Fossorier, "Quasicyclic low-density parity-check codes from circulant permutation matrices," IEEE Transactions on Information Theory, vol. 50, no. 8, pp. 1788-1793, 2004.

[20] J. Li, K. Liu, S. Lin, and K. Abdel-Ghaffar, "Decoding of quasicyclic LDPC codes with section-wise cyclic structure," in 2014 Information Theory and Applications Workshop (ITA), pp. 110, San Diego, CA, USA, 2014.

[21] Q. Huang, L. Tang, S. He, Z. Xiong, and Z. Wang, "Low-complexity encoding of quasi-cyclic codes based on Galois Fourier transform," IEEE Transactions on Communications, vol. 62, no. 6, pp. 1757-1767, 2014.

[22] R. M. Tanner, D. Sridhara, and T. E. Fuja, "A class of groupstructured LDPC codes," in Proceedings. International Symposium Communication Theory and Applications, Ambleside, U.K., 2001.

[23] X. Sun and I. B. Djordjevic, "LDPC codes derived from quasi-cyclic code design suitable for optical communications," in 2018 20th International Conference on Transparent Optical Networks (ICTON), pp. 1-4, Bucharest, Romania, 2018.

[24] T. J. Richardson and R. L. Urbanke, "Efficient encoding of lowdensity parity-check codes," IEEE Transactions on Information Theory, vol. 47, no. 2, pp. 638-656, 2001.

[25] E. Yeo, P. Pakzad, B. Nikolic, and V. Anantharam, "High throughput lowdensity parity-check decoder architectures," in GLOBECOM'01. IEEE Global Telecommunications Conference (Cat. No.01CH37270), pp. 3019-3024, San Antonio, TX, USA, 2001.

[26] M. Mansour and N. Shanbhag, "Turbo decoder architectures for low-density parity-check codes," in Global Telecommunications Conference, 2002. GLOBECOM '02. IEEE, pp. 13831388, Taipei, Taiwan, 2002. 